\title{
Features of acetabular labral tears on X-ray, magnetic resonance imaging and hip arthroscopy - the observational pilot study
}

\author{
Xi-Liang Shang', Jia-Wen Zhang', Ji-Wu Chen', Yun-Xia Li', Shi-Yi Chen ${ }^{1}$
}

1Department of Sports Medicine, Huashan Hospital, Fudan University, Shanghai, China 2Department of Radiology, Huashan Hospital, Fudan University, Shanghai, China

\author{
Submitted: 16 September 2011 \\ Accepted: 13 November 2011
}

Arch Med Sci 2013; 9, 2: 297-302

DOI: 10.5114 /aoms.2012.31305

Copyright $\odot 2013$ Termedia \& Banach

\section{Corresponding author:}

Shi-Yi Chen

Department of Sports Medicine

Huashan Hospital

Fudan University

12 Wu Lu Mu Qi Road

Shanghai 200040, China

Phone: +86 021-52888255

Fax: +86 021-62496020

E-mail: sychen0011@163.com

\begin{abstract}
Introduction: To analyze the X-ray and magnetic resonance imaging (MRI) features of acetabular labral tears, so as to improve the recognition of the disease. Material and methods: Imaging features of 19 patients with acetabular labral tears confirmed by arthroscopy were analyzed retrospectively. All cases were examined by X-ray. Fourteen patients underwent MRI examinations.

Results: Seventeen of the 19 patients had at least one osseous abnormality consistent with femoroacetabular impingement (FAI) under arthroscopy; among them 7 cases were cam impingement, 3 cases were pincer impingement and 7 cases were mixed impingement. X-ray manifestations: 12 of the 19 patients had FAl; among them 6 cases were cam impingement, 2 cases were pincer impingement and 4 cases were mixed impingement. Five cases showed various degrees of degenerations of hip joint. Two patients had no identifiable structural abnormalities. Magnetic resonance imaging showed various degrees of injury of labrum in 6 patients, joint effusion in 5 cases, and bone marrow edema of femoral head in 3 cases. Conclusions: The FAI has much to do with acetabular labral tears. X-ray examination is important for FAI diagnosis. Given its high sensitivity and accuracy, MRI is an effective preoperative tool for defining the location and extent of labral tears. Combined with X-ray imaging and clinical manifestation, MRI can provide a reliable basis for clinical diagnosis and treatment of acetabular labral tears.
\end{abstract}

Key words: acetabular labral tears, femoroacetabular impingement, magnetic resonance imaging, arthroscopy.

\section{Introduction}

The acetabular labrum is triangular in cross section, with the base attached to the acetabular rim and the apex being the free margin [1]. Its function is to deepen the acetabulum, making it more difficult for the head of the femur to slip out of place. Acetabular labral tears are a potential cause of hip pain, especially for young people [2]. These tears are associated with trauma, classic hip dysplasia, Legg-Calve-Perthes disease, and osteoarthritis (OA). McCarthy et al. performed a study in which 436 patients who had hip symptoms consisting of pain localized to the groin were enrolled. Two hundred and forty-one of the 436 patients (55.3\%) in the study group had 261 labral tears on arthroscopic examination [3]. Although lesions of the acetabular labrum were considered a rarity, the prevalence of labral lesions has become more related to the development of hip joint 
OA, which can seriously impair the life of people. Moreover, the rates of misdiagnosis and missed diagnosis were high. In order to further enhance the understanding of imaging characteristics of acetabular labral tears, X-ray and magnetic resonance imaging (MRI) findings of cases with arthroscopically proven acetabular labral tears in our hospital were retrospectively analyzed. We hope that this study can play an important role in clinical diagnosis, prognosis and assessment of the treatment effect.

\section{Material and methods}

\section{Study population}

We retrospectively evaluated 19 patients of arthroscopically acetabular labral tears who were admitted to the sports medical department of Huashan Hospital affiliated to Fudan University from July 2008 to July 2010. There were 9 males and 10 females (mean age: 40.9 years, 8 left hips and 11 right ones). The course of acetabular labral tears ranged from 1 month to 5 years, with an average of 22 months. All patients presented with a history of hip pain, characterized by pain in groin, trochanter or buttock. Among these cases, 4 cases had locked joint. The operations were performed by doctors who are specialized in hip arthroscopy. All patients received X-ray examination before the operation. Fourteen cases were examined by MRI.

\section{Imaging examination}

Nineteen cases underwent $\mathrm{X}$-ray examination before the operation. All of them had the frog-leg lateral view of the hip and anteroposterior pelvic (AP) radiograph. Examinations of plain MRI scan were performed on a Siemens Trio Magnetom Verio 3.OT MRI scanner. The MRI using T1-weighted imaging (T1WI), short TI inversion recovery (STIR) sequence and Fast Relaxation Fast Spin Echo sequence $\mathrm{T} 2$ weighted imaging with fat suppression (fs-FRFSE-T2) on axial and coronal sections was performed in 14 cases, using $4 \mathrm{~mm}$ slice thickness, with $1 \mathrm{~mm}$ interval.

\section{Definition of femoroacetabular impingement syndrome (FAl)}

\section{Arthroscopic appearances}

Cam impingement characterized by a nonspherical femoral head and an abnormal prominence of the head-neck junction. The prominence produces a cam effect as the prominence of the femoral head impinges against the associated region of the acetabulum when the femoral head rotates into the hip joint. Pincer impingement results from local or global over-coverage of the femoral head by the acetabulum with no abnormal sphericity of the femoral head. Any morphologic anomaly that results in relative deepening of the socket can lead to pincer impingement, including retroversion of the acetabulum and coxa profunda. The combination of cam and pincer impingement is mixed impingement.

\section{X-ray manifestations}

Cam impingement can be defined as $\alpha$ angle $>50^{\circ} \mathrm{C}$ or head-neck offset $<7.2 \mathrm{~mm}$ on the crosstable lateral view of the hip $[4,5]$. Pincer impingement can be defined as retroversion of the acetabulum or coxa profunda on the anteroposterior pelvic (AP) radiograph $[6,7]$. The acetabular retroversion exists when any part of the anterior acetabular wall is more lateral than the posterior wall in the proximal region of the acetabulum (the so-called crossover or figure-of-eight sign). Coxa profunda is present when the floor of the acetabular fossa contacts or overlaps the ilioischial line on the AP view. As mentioned above, the combination of cam and pincer impingement is mixed impingement.

\section{Image analysis}

The radiological data of 19 cases were collected and analyzed retrospectively by experienced doctors with high professional title. Measurement: (1) $\alpha$ angle: The angle was obtained by placing a circle around the oblique axial circumference of the femoral head, a line in the center of the femoral neck along its longitudinal axis, and by placing a second line that extended from the intersection of the first line and the center of the femoral head to the point where the osseous anterior femoral head intersected the circle [8]. (2) Femoral headneck offset: a line was drawn through the center of the long axis of the femoral neck (line 1). A parallel line was drawn through the anterior most aspect of the femoral neck (line 2) and another parallel line was drawn along the anterior aspect of the femoral head (line 3). The distance between lines 2 and 3 was the anterior femoral head-neck offset [5].

\section{Results}

\section{Arthroscopic appearances}

Seventeen of the 19 (17/19, 89.5\%) patients had at least one osseous abnormality consistent with FAl under arthroscopy. Among them 7 cases were cam impingement, 3 cases were pincer impingement and 7 cases were mixed impingement. Assessment of these arthrographs revealed that $68.4 \%$ of the acetabular labral tears were located most often in the anterosuperior part of the labrum ( $n=13,13 / 19)$, followed by the superior $(n=5)$, and posterosuperior $(n=1)$ parts of the labrum.

\section{X-ray manifestations}

According to the diagnostic criteria of FAI mentioned above, $\alpha$ angle $>50^{\circ} \mathrm{C}$ or head-neck offset 
$<7.2 \mathrm{~mm}$ was cam impingement, retroversion of the acetabulum or coxa profunda was pincer impingement and the combination of them was mixed impingement. Twelve of the 19 patients had FAI $(12 / 19,63.2 \%)$; among them 6 cases were cam impingement, 2 cases were pincer impingement and 4 cases were mixed impingement (Figures 1-3). Five cases showed various degrees of degenera- tions of the hip joint (such as joint space narrowing or acetabular rim hyperplasia, arthrosclerosis and so on). Two patients had no identifiable structural abnormalities.

\section{Magnetic resonance imaging manifestations}

On T1WI, the shape of the normal labrum was triangular with a sharp edge and was attached to
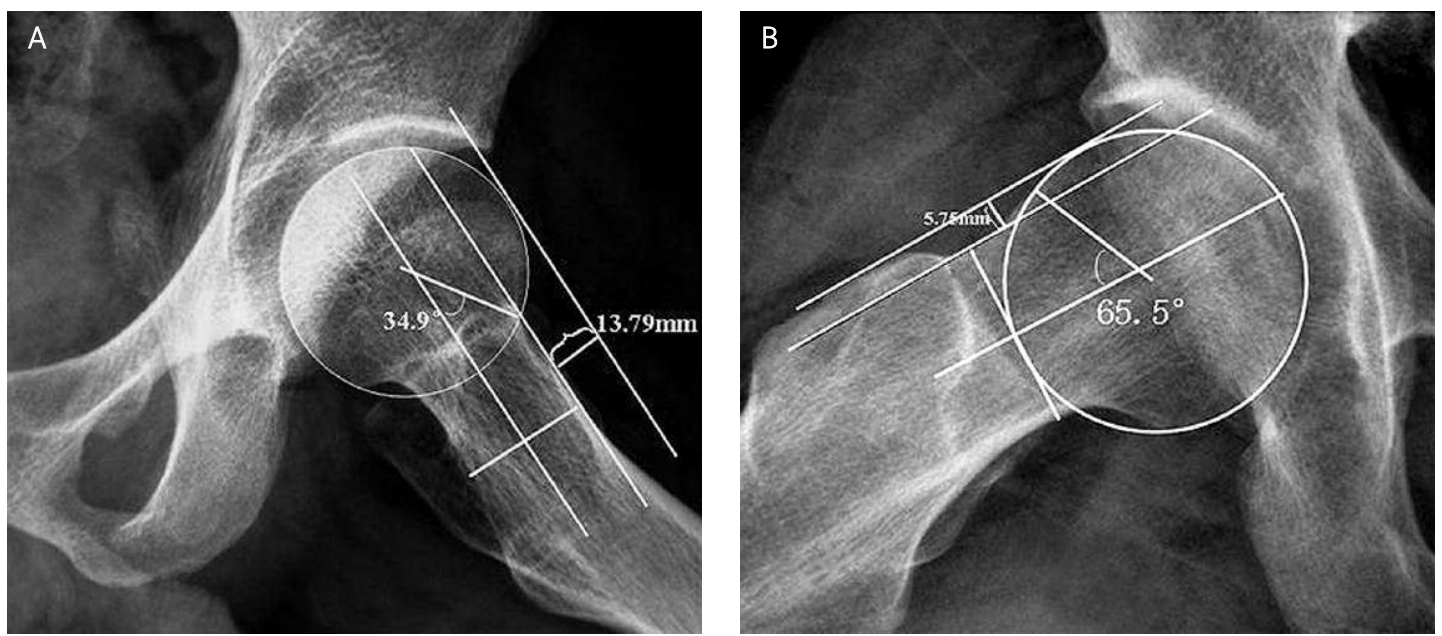

Figure 1. Frog-leg lateral radiograph of the normal and abnormal hip joint. A - Normal hip joint: $\alpha$ angle was $34.9^{\circ} \mathrm{C}$, femoral head-neck offset was $13.79 \mathrm{~mm}$. B - Abnormal hip joint (cam impingement): $\alpha$ angle was $65.5^{\circ} \mathrm{C}$ $\left(>50^{\circ} \mathrm{C}\right)$, femoral head-neck offset was $5.75 \mathrm{~mm}(<7.2 \mathrm{~mm})$
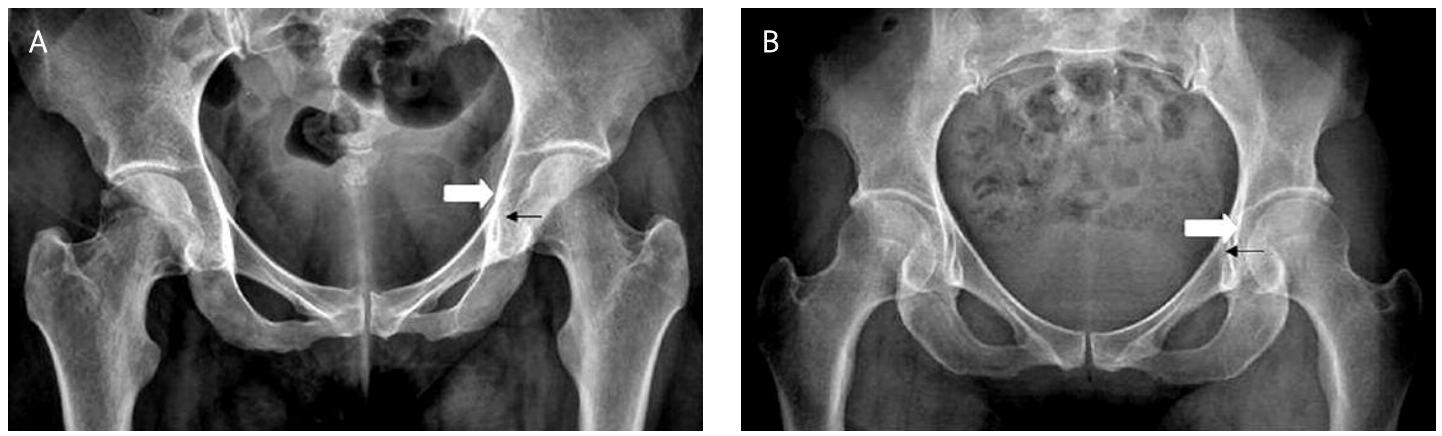

Figure 2. AP radiograph of the pelvis showed normal hip joint and coxa profunda, A - Normal hip joint, the acetabular floor (black arrow) lateral to the ilioischial line (white arrow). B - Coxa profunda, the acetabular floor (black arrow) medial to the ilioischial line (white arrow)
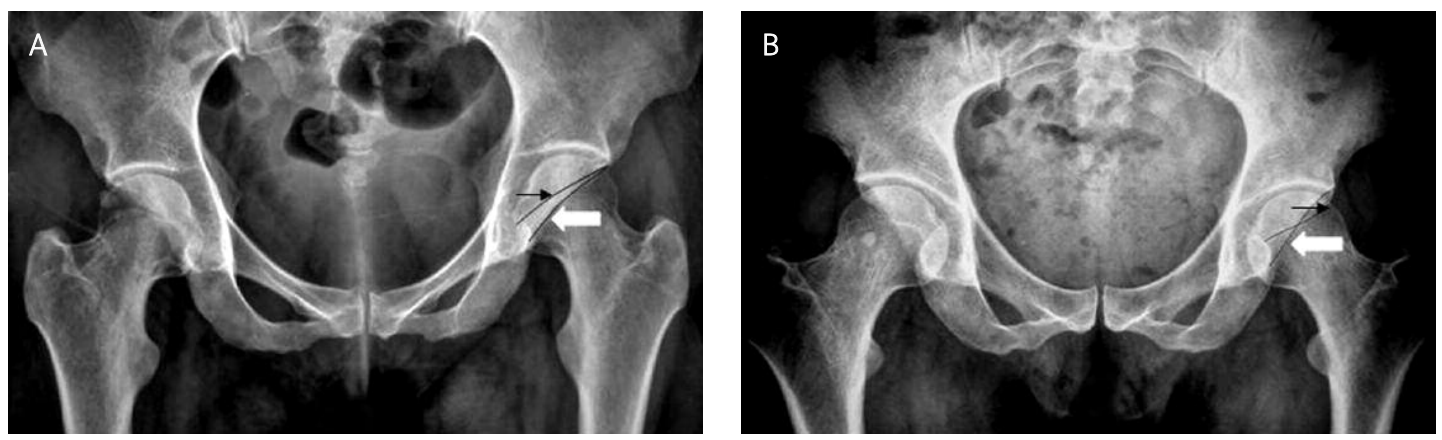

Figure 3. AP radiograph of the pelvis showed normal hip joint and acetabular retroversion. A - Normal hip joint, the anterior acetabular rim (black arrow) medial to posterior acetabular rim (white arrow); there was no crossing between them. B - Retroverted acetabula; the anterior acetabular rim (black arrow) is more lateral than the posterior acetabular rim (white arrow) (the so-called crossover or figure-of-eight sign) 
the acetabulum by the base. Homogeneous low signal intensity was observed in labral segments. Fourteen of the 19 patients were examined by MRI. Six patients $(6 / 14,42.9 \%)$ showed various degrees of labrum tears. On STIR, the labrum was irregular in shape and the signal intensity was inhomogeneous with spotted or patchy anomalous high intensity scattered around (Figure 4 B), or a linear high signal extending to the articular surface was demonstrated between the acetabular cartilage and the base of the labrum (Figure $4 \mathrm{C}$ ). Among cases, only 5 patients presented with joint effusion ( 5 of 14 cases, $35.7 \%$, Figure 4 D), and in only 3 cases was there bone marrow edema of the femoral head on fsFRFSE-T2 (Figure $4 \mathrm{E}$ ).

\section{Discussion}

Acetabular labral tears are increasingly recognized as a potential cause of hip pain. However, the disease lacks a specific clinical manifestation and we do not yet fully understand its imaging features. Therefore, the rates of misdiagnosis and missed diagnosis are high. Recently, the greater use of arthroscopy has led to a further understanding of this disease. Wenger et al. reported that 27 of 31 (87\%) acetabular labral tear patients had FAI [5]. In agreement with the literature report, we found that
17 of the 19 patients (89.5\%) had FAl under arthroscopy, which indicated that FAI had much to do with acetabular labral tears. Assessment of these arthrographs revealed that $68.4 \%$ (13 of 19 cases) of the acetabular labral tears were most often located in the anterosuperior part of the labrum, which suggested that the most common site of labrum tears was the anterosuperior labrum. The preferential involvement of the anterosuperior labrum may be due to FAl. Hip abnormalities in FAl limit motion, in particular flexion and internal rotation, as repetitive impact of the proximal femoral neck occurs against the acetabular labrum and/or its adjacent cartilage $[9,10]$.

The FAl occurs when the femoral head and acetabulum rub abnormally, resulting in damage to the articular cartilage and/or the labrum, as well as limited range of motion (ROM). It can be divided into three types: cam, pincer and mixed impingement. According to the reports of Notzli et al. and Wenger et al., an increase in $\alpha$ angle or a reduction in the superior femoral head-neck offset was suggested to be the cause of the cam impingement $[4,5]$. Notzli et al. used the $\alpha$ angle as a measurement of the femoral head-neck contour [4]. In 39 patients with clinical symptoms of FAl, a mean $\alpha$ angle of $74^{\circ} \mathrm{C}$ was found, compared with a mean $\alpha$ angle of $42^{\circ} \mathrm{C}$
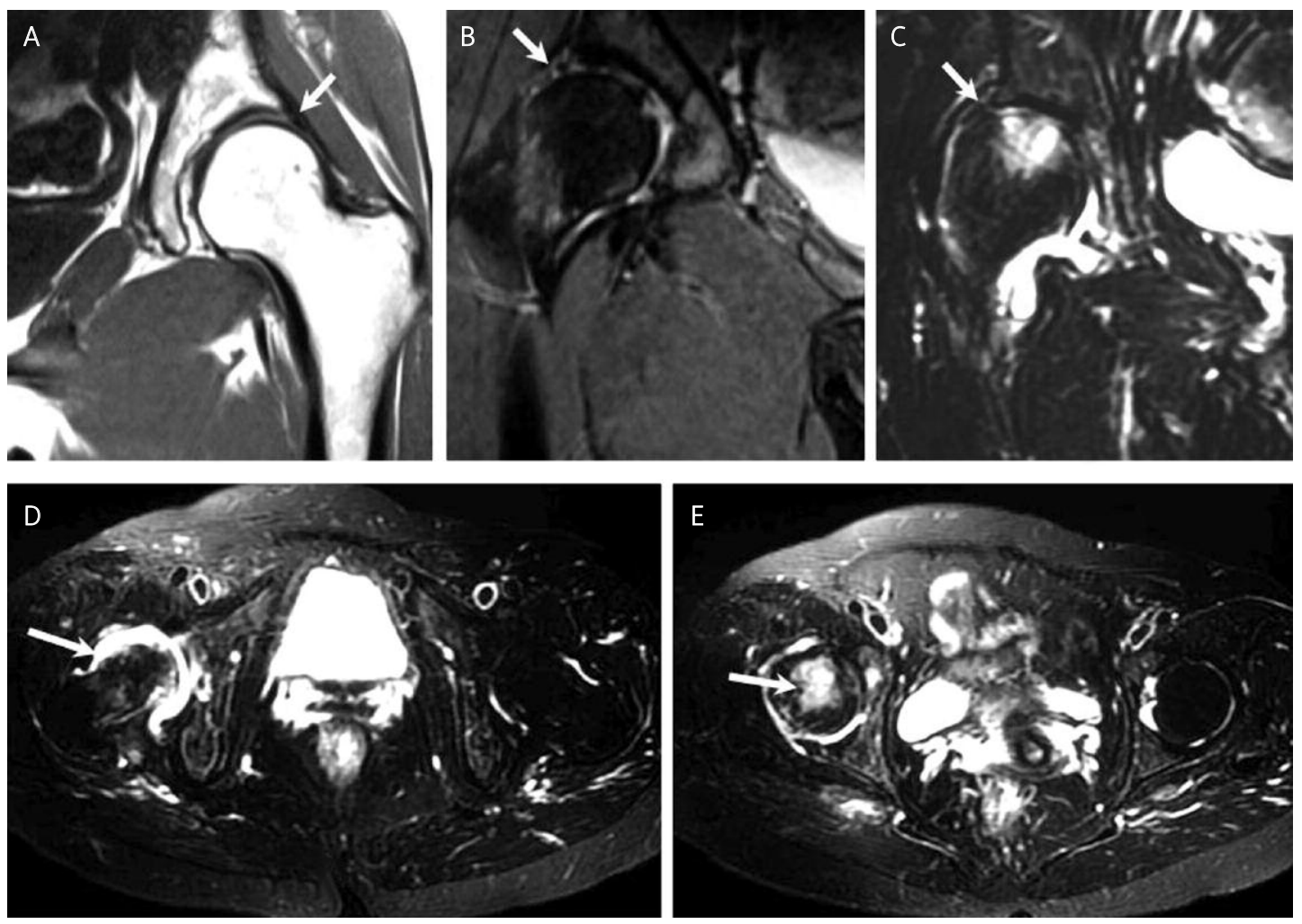

Figure 4. MRI manifestations of the acetabular labral tears. A-T1WI showed a normal acetabular labrum (white arrow), $B$ and C-STIR showed a region of increased signal in the labrum (white arrow) indicative of an acetabular labral tear, D-Fs-FRFSE-T2 showed high signal intensity in the articular cavity representing joint effusion, E - Fs-FRFSE-T2 showed high signal intensity in femoral head, indicating bone marrow edema 
for the control group. They proposed that an $\alpha$ angle of $>50^{\circ} \mathrm{C}$ may be an appropriate cross-sectional criterion in assessing for FAl. Wenger et al. found that the normal anterior femoral head-neck offset is $11.6 \mathrm{~mm}$ and they considered an offset of $<7.2 \mathrm{~mm}$ as abnormal [5]. Philippon et al. suggested that pincer impingement could be defined as retroversion of the acetabulum or coxa profunda on the AP radiograph [6]. According to the diagnostic criteria of FAl mentioned above, we analyzed the X-ray features of 19 patients and found that 12 cases had FAl (63.2\%); among them 6 cases were cam impingement, 2 cases were pincer impingement and 4 cases were mixed impingement. Depending on the arthroscopic findings, the accuracy of X-ray diagnosis in FAl reached $70.6 \%$ (12 of 17 cases). Considering that acetabular labral tears are closely related to FAl and FAI has typical X-ray manifestations, $X$-ray examination can provide an auxiliary proof in the diagnosis of acetabular labral tears by direct diagnosis of FAl.

Although X-ray examination is very important in the diagnosis of FAl, it cannot detect abnormal changes in the cartilage and labrum because of the lack of high resolution for soft tissue. The MRI is the preferred modality for the investigation of intraarticular hip pathology, as it provides exquisite resolution of the labrum, cartilage, and joint space as well as depicting the regional soft tissues. In our study, 14 cases were examined by MRI. Among them 6 patients (42.9\%) showed various degrees of labrum tears. On STIR, the labrum was irregular in shape and a high signal extending to the articular surface was found in it. Among all the cases, only 5 patients presented with joint effusion, and bone marrow edema of the femoral head was present in only 3 cases on fs-FRFSE-T2. We considered that the joint effusion might be due to wear or tear of the labrum and the bone marrow edema of the femoral head might be caused by FAl. The above indicates that labrum tears should be suspected if there is joint effusion or bone marrow edema.

There were a number of limitations in the present study. First, the number of patients is relatively small. A larger number of patients would increase the power of the study, allowing for more externally valid conclusions on the relationship between FAI and acetabular labral tears. Second, since this was a retrospective study, many patients with acetabular labral tears were likely not included in data analysis. It is unavoidable, however, since the patients were selected on the basis of arthroscopically confirmed acetabular labral tears. Third, not all patients underwent preoperative MRI, which means that the shape of the labrum was unable to be evaluated in every patient. There might have been selection bias.

In short, FAl has much to do with acetabular labral tears. X-ray examination is important for FAI diagnosis. The MRI is superior to X-ray in the diagnosis of acetabular labral tears, because of its specificity and the broad range of conditions which can be detected when radiographs are negative. Especially when used without contrast, MRI is straightforward and, of course, does not involve exposure to radiation. Familiarity with these structural abnormalities is important for early detection and diagnosis, which may impact development of an optimal treatment plan. However, it still has low accuracy for conventional MRI plain scan in the diagnosis of acetabular labral tears. In this study, we could only achieve an accuracy of $42.9 \%$. Magnetic resonance arthrography (MRA) is the combination of MRI with intra-articular injection of gadolinium-based contrast agents and is the test of choice for evaluation of the acetabular labrum. The meta-analysis performed by Smith et al., which encompassed a review of 19 papers, showed that both MRI and MRA had moderate sensitivity and specificity (sensitivity $66 \%, 87 \%$; specificity $79 \%, 64 \%$ ), and diagnostic accuracy of MRA appeared to be superior to MRI in detecting acetabular labral tears on receiver operating characteristic (ROC) curve interpretation [11]. However, whether or not MRA is superior to MRI in the diagnosis of acetabular labral tears requires further study.

\section{Acknowledgments}

Shang Xi-Liang and Zhang Jia-Wen contributed equally to this work.

This research was supported by the Young Science Foundation of Fudan University (No. 09FQ66).

\section{References}

1. Seldes RM, Tan V, Hunt J, Katz M, Winiarsky R, Fitzgerald $\mathrm{RH}$ Jr. Anatomy, histologic features, and vascularity of the adult acetabular labrum. Clin Orthop Relat Res 2001; 382: 232-40.

2. Hickman JM, Peters CL. Hip pain in the young adult: diagnosis and treatment of disorders of the acetabular labrum and acetabular dysplasia. Am J Orthop 2001; 30: 459-67.

3. McCarthy JC, Noble PC, Schuck MR, Wright J, Lee J. The Otto E. Aufranc Award: the role of labral lesions to development of early degenerative hip disease. Clin Orthop Relat Res 2001; 393: 25-37.

4. Nötzli HP, Wyss TF, Stoecklin CH, Schmid MR, Treiber K, Hodler J. The contour of the femoral head-neck junction as a predictor for the risk of anterior impingement. J Bone Joint Surg Br 2002; 84: 556-60.

5. Wenger DE, Kendell KR, Miner MR, Trousdale RT. Acetabular labral tears rarely occur in the absence of bony abnormalities. Clin Orthop Relat Res 2004; 426: 145-50.

6. Philippon MJ, Stubbs AJ, Schenker ML, Maxwell RB, Ganz R, Leunig M. Arthroscopic management of femoroacetabular impingement: osteoplasty technique and literature review. Am J Sports Med 2007; 35: 1571-80.

7. Reynolds D, Lucas J, Klaue K. Retroversion of the acetabulum. A cause of hip pain. J Bone Joint Surg Br 1999; 81: 281-8.

8. Beall DP, Sweet CF, Martin HD, et al. Imaging findings of femoroacetabular impingement syndrome. Skeletal Radiol 2005; 34: 691-701. 
9. Ganz R, Parvizi J, Beck M, Leunig M, Nötzli H, Siebenrock KA. Femoroacetabular impingement: a cause for osteoarthritis of the hip. Clin Orthop Relat Res 2003; 417: 112-20.

10. Leunig M, Podeszwa D, Beck M, Werlen S, Ganz R. Magnetic resonance arthrography of labral disorders in hips with dysplasia and impingement. Clin Orthop Relat Res 2004; 418: 74-80.

11. Smith TO, Hilton G, Toms AP, Donell ST, Hing CB. The diagnostic accuracy of acetabular labral tears using magnetic resonance imaging and magnetic resonance arthrography: a meta-analysis. Eur Radiol 2011; 21: 863-74. 